\section{Probenverarbeitung, serielle}

W. G. Guder

München, Deutschland

Englischer Begriff batchwise sample processing

Definition Die Bearbeitung eintreffender Proben ( $\triangleright$ Probe) zur Untersuchung erfolgt in Serien, Gruppen oder Reihen. Gegensatz zu kontinuierlicher Probenbearbeitung.

Beschreibung Im Bearbeitungsprozess von Proben, die ein Laboratorium erreichen, geht man traditionell seriell vor, d. h. man lässt mehrere Proben zusammenkommen, bevor der nächste Schritt getan wird. Dies umfasst zum Beispiel die Zentrifugation, die Übernahme von Proben in ein analytisches System oder einen Arbeitsplatz und die $>$ Messung an einem Analysegerät. Nachdem dieser Vorgang als Quelle und bedeutender Faktor der gesamten Bearbeitungszeit erkannt wurde, hat man ihn gegenüber der kontinuierlichen Bearbeitung von Proben als nachteilig erkannt und mithilfe von präanalytischen Straßen versucht, die serielle Bearbeitung durch kontinuierliche Bearbeitung zu beschleunigen, wie es im traditionellen Notfalllabor schon immer versucht wurde.

\section{Literatur}

Godolphin W, Bodtker K, Wilson L (1992) Simulation modelling: a tool to help predict the impact of automation in clinical laboratories. Lab Robot Autom 4:249-255 